\title{
Dual Field Formulation of Quantum Integrable Models
}

\author{
V. E. Korepin \\ Leningrad Department of Steklov Mathematical Institute, Fontanka 27, \\ SU-191011, Leningrad, USSR
}

\begin{abstract}
Equal time correlators are studied in completely integrable models. The main example is the quantum non-linear Schrödinger equation. Introduction of an auxiliary Fock space permits us to represent the generating functional of correlators in the form of a determinant of the integral operator.
\end{abstract}

\section{Introduction}

The Algebraic Bethe Ansatz [1] permits us to advance in the analysis of completely integrable quantum models. Correlation functions are of special interest. The approach of papers [2-6] will be developed here. We shall illustrate new results in correlation functions on the example of the quantum non-linear Schrödinger equation (another name of the model is the one-dimensional Bose gas). The Hamiltonian $H$ and commutation relations of the model are:

$$
\begin{gathered}
H=\int_{0}^{L} d x\left(\partial_{x} \psi^{+} \partial_{x} \psi+c \psi^{+} \psi^{+} \psi \psi\right), \quad c>0, \\
{\left[\psi(x), \psi^{+}(y)\right]=\delta(x-y), \quad[\psi(x), \psi(y)]=\left[\psi^{+}(x), \psi^{+}(y)\right]=0 .}
\end{gathered}
$$

Here $\psi$ is a canonical Bose field, $L$ is the length of periodic box and $c$ is a coupling constant. The Fock space in which the quantum fields $\psi, \psi^{+}$act will be called the initial Fock space, because later there will appear another auxiliary Fock space. The algebraic vacuum $|0\rangle$ in the initial Fock space is defined in the standard way:

$$
\psi(x)|0\rangle=0 .
$$

The dual algebraic vacuum $\langle 0|=| 0\rangle^{+}$is also important

$$
\langle 0| \psi^{+}(x)=0, \quad\langle 0 \mid 0\rangle=1 .
$$

We shall consider the ground state of the model in the sector with fixed density $D$ and calculate correlators for this case. The correlators of conserving currents are of main interest for us. The simplest example is:

$$
\left\langle\psi^{+}(x) \psi(x) \psi^{+}(0) \psi(0)\right\rangle, \quad x>0 .
$$


In the next section we shall introduce the generating functional of correlation functions.

\section{Trace Identities}

The foundation of the solution of the model both in quantum and classical cases is the Lax representation. The transition matrix $T(x, y / \lambda)$ plays an important role in the inverse scattering method. In the classical case it is defined by the differential equation and initial data:

$$
\left[\frac{\partial}{\partial x}+\frac{i \lambda}{2} \sigma_{3}+\mathbf{q}(x)\right] T(x, y \mid \lambda)=0, \quad T(y, y \mid \lambda)=\left(\begin{array}{ll}
1 & 0 \\
0 & 1
\end{array}\right) .
$$

The transition matrix is a $2 \times 2$ matrix, the value $\lambda$ is a spectral parameter, $\sigma_{3}$ is the Pauli matrix $\sigma_{3}=\operatorname{diag}(1,-1)$, and the potential $\mathbf{q}(x)$ is equal to

$$
\mathbf{q}(x)=\sqrt{c}\left(\begin{array}{cc}
0, & i \psi^{+}(x) \\
-i \psi(x), & 0
\end{array}\right) .
$$

The transition matrix for period $L$ is called the monodromy matrix $T(\lambda)$ :

$$
T(\lambda)=T(L, 0 \mid \lambda) .
$$

The trace of the monodromy matrix $\tau(\lambda)$ is important,

$$
\tau(\lambda)=\operatorname{tr} T(\lambda) .
$$

In the quantum case the transition matrix $T(x, y \mid \lambda)$ can be constructed as follows. In the expression for classical $T(x, y \mid \lambda)$, move all $\psi$ to the right and all $\psi^{+}$to the left, then replace them by the quantum operators:

$$
T(x, y \mid \lambda)_{q}=: T(x, y \mid \lambda)_{c l}: .
$$

This permits us to solve the problem of trace identities in the quantum case. Coefficients of asymptotic decomposition of the transition matrix at $\lambda \rightarrow i \infty$ are reduced to conserving currents. To write down first a few coefficients, let us introduce notations:

$$
\begin{aligned}
& T_{1}(\lambda)=T(x, 0 \mid \lambda)=\left(\begin{array}{ll}
A_{1}(\lambda), & B_{1}(\lambda) \\
C_{1}(\lambda), & D_{1}(\lambda)
\end{array}\right), \quad x>0, \\
& a_{1}(\lambda)=\exp \{-i \lambda x / 2\}, \quad d_{1}(\lambda)=\exp \{i \lambda x / 2\} .
\end{aligned}
$$

At $\lambda \rightarrow i \infty$ the values $A_{1}(\lambda)$ and $D_{1}(\lambda)$ behave as follows:

$$
\begin{aligned}
A_{1}(\lambda)= & a_{1}(\lambda)\left\{1+\frac{i c}{\lambda} \int_{0}^{x} \psi^{+}(z) \psi(z) d z+\frac{c}{\lambda^{2}} \psi^{+}(0) \psi(0)\right. \\
& +\frac{c}{\lambda^{2}} \int_{0}^{x} d z \psi^{+}(z) \partial_{z} \psi(z) \\
& \left.-\frac{c^{2}}{2 \lambda^{2}} \int_{0}^{x} d z_{1} \int_{0}^{x} d z_{2} \psi^{+}\left(z_{1}\right) \psi^{+}\left(z_{2}\right) \psi\left(z_{1}\right) \psi\left(z_{2}\right)+O\left(\frac{1}{\lambda^{3}}\right)\right\}, \\
& D_{1}(\lambda)=\frac{-c}{\lambda^{2}} a_{1}(\lambda)\left\{\psi^{+}(0) \psi(x)+O\left(\frac{1}{\lambda}\right)\right\} .
\end{aligned}
$$


As far as we operate with the asymptotic expansion in $1 / \lambda$ we can replace $d_{1}(\lambda)$ by zero:

$$
\exp \{i \lambda x / 2\}=d_{1}(\lambda) \Rightarrow 0 ; \quad \lambda \rightarrow i \infty .
$$

The mean value of $A_{1}(\lambda)$ with respect to the ground state of the model is the generating functional of correlators

$$
\left\langle A_{1}(\mu)\right\rangle \text {. }
$$

For example correlator (1.4) can be expressed as follows:

$$
\frac{\partial^{2}}{\partial x^{2}} \frac{\left\langle A_{1}(\mu)\right\rangle}{a_{1}(\mu)}=\frac{-c^{2}}{\mu^{2}}\left\langle\psi^{+}(x) \psi(x) \psi^{+}(0) \psi(0)\right\rangle+O\left(\frac{1}{\mu^{3}}\right), \quad \mu \rightarrow i \infty, x>0 .
$$

Here we replace $\lambda$ by $\mu$ because it will be more convenient later. In Sect. 9 it will be shown that $\left\langle A_{1}(\mu)\right\rangle / a_{1}(\mu)$ is equal to the determinant of the integral operator (in the thermodynamic limit). The kernel of this integral operator will be a quantum operator in the auxiliary Fock space.

\section{Two-Site Generalized Model}

To investigate correlation functions in the framework of the quantum inverse scattering method it is convenient [4-6] to consider the two-site generalized model. Let us regard two matrices $T_{1}(\lambda)$ and $T_{2}(\lambda)$ (the subindex is the number of the site)

$$
T_{1,2}(\lambda)=\left(\begin{array}{ll}
A_{1,2}(\lambda) & B_{1,2}(\lambda) \\
C_{1,2}(\lambda) & D_{1,2}(\lambda)
\end{array}\right)
$$

The matrix elements of $T_{1}(\lambda)$ and $T_{2}(\lambda)$ are quantum operators in two different Fock spaces (both initial). The vectors

$$
\prod_{j=1}^{N} B_{1}\left(\lambda_{j}\right)|0\rangle_{1}
$$

form the basis in the first Fock space, and the vectors

$$
\prod_{j=1}^{N} B_{2}\left(\lambda_{j}\right)|0\rangle_{2}
$$

are the basis in the second Fock space. Commutation relations of $A, B, C, D$ are given by the $R$ matrix:

$$
R(\lambda, \mu)\left(T_{1}(\lambda) \otimes T_{1}(\mu)\right)=\left(T_{1}(\mu) \otimes T_{1}(\lambda)\right) R(\lambda, \mu) .
$$

It is a $4 \times 4$ matrix:

$$
R(\lambda, \mu)=\left(\begin{array}{cccc}
f(\mu, \lambda) & 0 & 0 & 0 \\
0 & g(\mu, \lambda) & 1 & 0 \\
0 & 1 & g(\mu, \lambda) & 0 \\
0 & 0 & 0 & f(\mu, \lambda)
\end{array}\right)
$$


Here $f$ and $g$ are equal to:

$$
f(\mu, \lambda)=\left(\frac{\mu-\lambda+i c}{\mu-\lambda}\right), \quad g(\mu, \lambda)=\left(\frac{i c}{\mu-\lambda}\right) .
$$

The function

$$
h(\mu, \lambda)=\frac{f(\mu, \lambda)}{g(\mu, \lambda)}=\left(\frac{\mu-\lambda+i c}{i c}\right)
$$

will also be useful. The matrix $T_{2}$ satisfies the same relation (3.4). Matrix elements of $T_{1}$ commute with matrix elements of $T_{2}$. Algebraic vacuums $|0\rangle_{1,2}$ have the following properties:

$$
\begin{gathered}
C_{1,2}(\lambda)|0\rangle_{1,2}=0, \quad A_{1,2}(\lambda)|0\rangle_{1,2}=a_{1,2}(\lambda)|0\rangle_{1,2}, \\
D_{1,2}(\lambda)|0\rangle_{1,2}=d_{1,2}(\lambda)|0\rangle_{1,2} .
\end{gathered}
$$

Here $a_{1,2}(\lambda)$ and $d_{1,2}(\lambda)$ are four arbitrary complex valued functions. We shall vary them later. The matrix product of $T_{2}$ and $T_{1}$

$$
T(\lambda)=T_{2}(\lambda) \cdot T_{1}(\lambda)=\left(\begin{array}{ll}
A(\lambda) & B(\lambda) \\
C(\lambda) & D(\lambda)
\end{array}\right)
$$

is called a monodromy matrix. It also satisfies (3.4). Its algebraic vacuum is equal to

$$
|0\rangle=|0\rangle_{1} \times|0\rangle_{2} \text {. }
$$

Relations

$$
\begin{gathered}
C(\lambda)|0\rangle=0, \quad A(\lambda)|0\rangle=a(\lambda)|0\rangle, \quad D(\lambda)|0\rangle=d(\lambda)|0\rangle, \\
a(\lambda)=a_{1}(\lambda) a_{2}(\lambda), \quad d(\lambda)=d_{1}(\lambda) d_{2}(\lambda)
\end{gathered}
$$

are valid. The basis

$$
\prod_{j=1}^{N} B\left(\lambda_{j}\right)|0\rangle
$$

can be expressed in terms of (3.2) and (3.3),

$$
\begin{aligned}
\prod_{j=1}^{N} B\left(\lambda_{j}\right)|0\rangle= & \sum_{\left\{\lambda_{3}=\left\{\lambda_{\mathrm{I}}\right\} \cup\left\{\lambda_{\mathrm{II}}\right\}\right.} \prod_{\mathrm{I}} \prod_{\mathrm{II}} a_{2}\left(\lambda_{\mathrm{I}}\right) d_{1}\left(\lambda_{\mathrm{II}}\right) f\left(\lambda_{\mathrm{I}}, \lambda_{\mathrm{II}}\right) \\
& \times B_{2}\left(\lambda_{\mathrm{II}}\right)|0\rangle_{2} B_{1}\left(\lambda_{\mathrm{I}}\right)|0\rangle_{1} .
\end{aligned}
$$

Here summation is over partitions of the set $\left\{\lambda_{j}\right\}$ into two disjoint subsets $\left\{\lambda_{I}\right\}$ and $\left\{\lambda_{\mathrm{II}}\right\}$. A similar formula is valid for the dual basis:

$$
\begin{aligned}
\langle 0| \prod_{j=1}^{N} C\left(\lambda_{j}\right)= & \sum_{\{\lambda\}=\left\{\lambda_{\mathrm{I}}\right\} \cup\left\{\lambda_{\mathrm{II}}\right\}} \prod_{\mathrm{I}} \prod_{\mathrm{II}}\langle 0| C_{1}\left(\lambda_{\mathrm{I}}\right)_{2}\langle 0| C_{2}\left(\lambda_{\mathrm{II}}\right) \\
& \times f\left(\lambda_{\mathrm{II}}, \lambda_{\mathrm{I}}\right) a_{1}\left(\lambda_{\mathrm{II}}\right) d_{2}\left(\lambda_{\mathrm{I}}\right) .
\end{aligned}
$$


This permits to express matrix element of $A_{1}(\mu)$ in the form:

$$
\begin{aligned}
\langle 0| & \prod_{j=1}^{N} C\left(\lambda_{j}^{c}\right) A_{1}(\mu) \prod_{j=1}^{N} B\left(\lambda_{j}^{B}\right)|0\rangle \\
= & \sum_{1}\left\langle 0\left|\prod_{\mathrm{I}} C_{1}\left(\lambda_{\mathrm{I}}^{c}\right) A_{1}(\mu) \prod_{\mathrm{I}} B_{1}\left(\lambda_{\mathrm{I}}^{B}\right)\right| 0\right\rangle_{12}\left\langle 0\left|\prod_{\mathrm{II}} C_{2}\left(\lambda_{\mathrm{II}}^{c}\right) \prod_{\mathrm{II}} B_{2}\left(\lambda_{\mathrm{II}}^{B}\right)\right| 0\right\rangle_{2} \\
& \times \prod_{\mathrm{I}} \prod_{\mathrm{II}} a_{2}\left(\lambda_{\mathrm{I}}^{B}\right) d_{2}\left(\lambda_{\mathrm{I}}^{c}\right) a_{1}\left(\lambda_{\mathrm{II}}^{c}\right) d_{1}\left(\lambda_{\mathrm{II}}^{B}\right) f\left(\lambda_{\mathrm{I}}^{B}, \lambda_{\mathrm{II}}^{B}\right) f\left(\lambda_{\mathrm{II}}^{c}, \lambda_{\mathrm{I}}^{c}\right) .
\end{aligned}
$$

Here summation is over two independent partitions $\left\{\lambda^{B}\right\}=\left\{\lambda_{\mathrm{I}}^{B}\right\} \cup\left\{\lambda_{\mathrm{II}}^{B}\right\}$ and $\left\{\lambda^{c}\right\}$ $=\left\{\lambda_{\mathrm{I}}^{c}\right\} \cup\left\{\lambda_{\mathrm{II}}^{c}\right\}$, except from one condition: number of elements in $\left\{\lambda_{\mathrm{I}}^{c}\right\}$ is equal to the number of elements in $\left\{\lambda_{\mathrm{I}}^{B}\right\}\left(\#\left\{\lambda_{\mathrm{I}}^{c}\right\}=\#\left\{\lambda_{\mathrm{I}}^{B}\right\}\right)$.

The eigenfunctions of transfermatrix $\tau(\mu)=A(\mu)+D(\mu)$ (and the Hamiltonian) are given by (3.12) in the case, if the system of transcendental equations are satisfied:

$$
\frac{a\left(\lambda_{n}\right)}{d\left(\lambda_{n}\right)} \prod_{k=1}^{N} \frac{h\left(\lambda_{n}, \lambda_{k}\right)}{h\left(\lambda_{k}, \lambda_{n}\right)}=(-1)^{N-1}
$$

The dual eigenfunction is:

$$
\langle 0| \prod_{j=1}^{N} C\left(\lambda_{j}\right)
$$

with the same set of $\lambda_{j}$.

Nonlinear Schrödinger equation is the special case of two-site generalized model. We must put

$$
\begin{array}{ccc}
a(\lambda)=\exp \{-i \lambda L / 2\}, & d(\lambda)=\exp \{i \lambda L / 2\}, \quad L>0, \\
a_{1}(\lambda)=\exp \{-i \lambda x / 2\}, & d_{1}(\lambda)=\exp \{i \lambda x / 2\}, \quad x>0, \\
a_{2}(\lambda)=\exp \{i \lambda(x-L) / 2\}, & d_{2}(\lambda)=\exp \{i \lambda(L-x) / 2\}, \quad L>x .
\end{array}
$$

In this case $T_{1}(\lambda)$ from (3.1) will turn into $T_{1}(\lambda)$ from (2.6), the matrix $T_{2}(\lambda)$ from (3.1) will turn into $T(L, x / \lambda)$ which is a special case of (2.5). Monodromy matrix (3.9) will become the monodromy matrix of the nonlinear Schrödinger equation (2.3).

In the next section we shall see that

$$
\left\langle 0\left|\prod_{j=1}^{N} C\left(\lambda_{j}^{c}\right) A(\mu) \prod_{j=1}^{N} B\left(\lambda_{j}^{B}\right)\right| 0\right\rangle
$$

is reduced to scalar product:

$$
\left\langle 0\left|\prod_{j=1}^{N} C\left(\lambda_{j}^{c}\right) \prod_{j=1}^{N} B\left(\lambda_{j}^{B}\right)\right| 0\right\rangle .
$$

In this way we see that (3.15) reduce matrix element of $A_{1}(\mu)$ to scalar products.

\section{Scalar Products}

The values

$$
\mathscr{S}_{N}=\left\langle 0\left|\prod_{j=1}^{N} C\left(\lambda_{j}^{c}\right) \prod_{j=1}^{N} B\left(\lambda_{j}^{B}\right)\right| 0\right\rangle=\mathscr{S}_{N}([a(\lambda), d(\lambda)])
$$


are called scalar products. (Here $N$ is the number of the particles.) They were studied in [2], they are functionals of two arbitrary functions $a(\lambda)$ and $d(\lambda)$. The matrix element of $A(\mu)$ :

$$
\left\langle 0\left|\prod_{j=1}^{N} C\left(\lambda_{j}^{c}\right) A(\mu) \prod_{j=1}^{N} B\left(\lambda_{j}^{B}\right)\right| 0\right\rangle=\langle A(\mu)\rangle[a(\lambda), d(\lambda)]
$$

also depends on two arbitrary functions $a(\lambda)$ and $d(\lambda)$. Now we shall see that (4.1) and (4.2) are related, if they are evaluated for different functions $a(\lambda)$ and $d(\lambda)$ :

$$
\left\langle 0\left|\prod_{j=1}^{N} C\left(\lambda_{j}^{c}\right) A(\mu) \prod_{j=1}^{N} B\left(\lambda_{j}^{B}\right)\right| 0\right\rangle^{I}=\left\langle 0\left|\prod_{j=1}^{N} C\left(\lambda_{j}^{c}\right) \prod_{j=1}^{N} B\left(\lambda_{j}^{B}\right)\right| 0\right\rangle a(\mu) .
$$

Index $I$ means that the left-hand side is evaluated for functions $a^{I}(\lambda)$ and $d^{I}(\lambda)$, and the right-hand side is evaluated for $a(\lambda)$ and $d(\lambda)$. Their relation is

$$
a^{I}(\lambda)=a(\lambda) ; \quad d^{I}(\lambda)=d(\lambda) f^{-1}(\mu, \lambda) .
$$

Let us formulate the theorem

Theorem 1. The values (4.1) and (4.2) are related by the following formula

$$
\langle A(\mu)\rangle\left[a^{I}(\lambda), d^{I}(\lambda)\right]=a(\mu) \mathscr{S}_{N}[a(\lambda), d(\lambda)]
$$

under the condition that $d^{I}(\mu)=0$.

It can be proved by means of the algebraic Bethe Ansatz $[2,3,6]$. It will be used in Sect. 8 for calculation of the generating functional of correlators.

Now let us present a recursion formula for scalar products, which will play an extremely important role below.

Theorem 2. The scalar products $\mathscr{S}_{N}$ and $\mathscr{S}_{N-1}$ are related by the following formula:

$$
\begin{aligned}
\mathscr{S}_{N}([a(\lambda), d(\lambda)])= & a\left(\lambda_{1}^{c}\right) \sum_{n=1}^{N} d\left(\lambda_{n}^{B}\right) g\left(\lambda_{1}^{c}, \lambda_{n}^{B}\right) \prod_{j \neq 1} g\left(\lambda_{1}^{c}, \lambda_{j}^{c}\right) \\
& \times \prod_{k \neq n} g\left(\lambda_{k}^{B}, \lambda_{n}^{B}\right) \mathscr{S}_{N-1}\left(\left[a(\lambda) h\left(\lambda, \lambda_{n}^{B}\right), d(\lambda) h\left(\lambda_{1}^{c}, \lambda\right)\right]\right) \\
& +d\left(\lambda_{1}^{c}\right) \sum_{n=1}^{N} a\left(\lambda_{n}^{B}\right) g\left(\lambda_{n}^{B}, \lambda_{1}^{c}\right) \prod_{j \neq n} g\left(\lambda_{n}^{B}, \lambda_{j}^{B}\right) \prod_{k \neq 1} g\left(\lambda_{k}^{c}, \lambda_{1}^{c}\right) \\
& \times \mathscr{S}_{N-1}\left(\left[a(\lambda) h\left(\lambda, \lambda_{1}^{c}\right), d(\lambda) h\left(\lambda_{n}^{B}, \lambda\right)\right]\right) .
\end{aligned}
$$

In $\mathscr{S}_{N-1}$ the set $\left\{\lambda^{B}\right\}$ is obtained from the left-hand side by removing $\lambda_{n}^{B}$, and set $\left\{\lambda^{c}\right\}$ by removing $\lambda_{1}^{c}(h(\lambda, \mu)=(\lambda-\mu+i c) / i c)$.

Proof can be given by the techniques of papers $[2,7]$.

I would like to emphasize that (4.6) and

$$
\mathscr{S}_{0}=\langle 0 \mid 0\rangle=1
$$

fix $\mathscr{S}_{N}$ in a unique way. In the next section we shall solve the recursion relation (4.6) by means of quantum operators in the auxiliary Fock space. 


\section{Dual Quantum Field Theory}

To solve the recursion relation (4.6), let us introduce auxiliary quantum fields in auxiliary Fock space. At first two coordinates $q_{A}(\lambda), q_{D}(\lambda)$ and two momenta $\pi_{D}(\lambda), \pi_{A}(\lambda)$ will be introduced. Their commutation relations are

$$
\left[\pi_{D}(\lambda), q_{D}(\mu)\right]=\ln h(\lambda, \mu), \quad\left[\pi_{A}(\lambda), q_{A}(\mu)\right]=\ln h(\mu, \lambda) .
$$

Here $h(\lambda, \mu)=(\lambda-\mu+i c) / i c$, and $\ln h(\lambda, \lambda)=0$. Other commutators are equal to zero. Vacuum in the auxiliary Fock space also will be denoted by $|0\rangle$. Momenta annihilate it,

$$
\pi_{D}(\lambda)|0\rangle=0, \quad \pi_{A}(\lambda)|0\rangle=0 .
$$

The dual vacuum $\langle 0|$ is the eigenfunction for the coordinates

$$
\langle 0| q_{A}(\lambda)=\ln a(\lambda)\langle 0| ; \quad\langle 0| q_{D}(\lambda)=\ln d(\lambda)\langle 0|, \quad\langle 0 \mid 0\rangle=1 .
$$

Auxiliary quantum fields $\Phi_{A}(\lambda)$ and $\Phi_{D}(\lambda)$ are defined as follows:

$$
\Phi_{A}(\lambda)=q_{A}(\lambda)+\pi_{D}(\lambda) ; \quad \Phi_{D}(\lambda)=q_{D}(\lambda)+\pi_{A}(\lambda) .
$$

It is interesting to note that they commute:

$$
\left[\Phi_{A}(\lambda), \Phi_{D}(\mu)\right]=\left[\Phi_{A}(\lambda), \Phi_{A}(\mu)\right]=\left[\Phi_{D}(\lambda), \Phi_{D}(\mu)\right]=0 .
$$

Let us define now the $N \times N$ matrix $S:(g(\lambda, \mu)=i c /(\lambda-\mu))$,

$$
S_{j k}=\frac{g\left(\lambda_{j}^{c}, \lambda_{k}^{B}\right)}{h\left(\lambda_{j}^{c}, \lambda_{k}^{B}\right)} \exp \left\{\Phi_{A}\left(\lambda_{j}^{c}\right)+\Phi_{D}\left(\lambda_{k}^{B}\right)\right\}+\frac{g\left(\lambda_{k}^{B}, \lambda_{j}^{c}\right)}{h\left(\lambda_{k}^{B}, \lambda_{j}^{C}\right)} \exp \left\{\Phi_{A}\left(\lambda_{k}^{B}\right)+\Phi_{D}\left(\lambda_{j}^{c}\right)\right\} .
$$

The determinant of this matrix is well defined (all matrix elements commute) $\operatorname{det}_{N} S$ (the subscript $N$ means dimension of the matrix). The vacuum mean value of this determinant is important:

$$
\left\langle 0\left|\operatorname{det}_{N} S\right| 0\right\rangle \text {. }
$$

To calculate it one must use commutation relations (5.1) to put $\operatorname{det}_{N} S$ in normal order (all $\pi$ to the right, all $q$ to the left). The mean value (5.7) is proportional to the scalar product (4.1).

Theorem 3. The scalar product $\mathscr{S}_{N}$ is equal to:

$$
\left\langle 0\left|\prod_{j=1}^{N} C\left(\lambda_{j}^{c}\right) \prod_{j=1}^{N} B\left(\lambda_{j}^{B}\right)\right| 0\right\rangle=\prod_{j>k} g\left(\lambda_{j}^{c}, \lambda_{k}^{c}\right) g\left(\lambda_{k}^{B}, \lambda_{j}^{B}\right)\left\langle 0\left|\operatorname{det}_{N} S\right| 0\right\rangle .
$$

Proof. One should develop $\operatorname{det}_{N} S$ in the first line (associated with $\lambda_{1}^{c}$ ) and note that replacement of the dual vacuum $\langle 0|$ by $\langle 0| \exp \left\{\pi_{D}\left(\lambda_{D}\right)+\pi_{A}\left(\lambda_{A}\right)\right\}$ means replacement of $a(\mu) \rightarrow a(\mu) h\left(\mu, \lambda_{A}\right), d(\mu) \rightarrow d(\mu) h\left(\lambda_{D}, \mu\right)$. Obtained in this way the relation of $\mathscr{S}_{N}$ and $\mathscr{S}_{N-1}$ will coincide with (4.6). The relation $\langle 0 \mid 0\rangle=1$ completes the proof.

\section{Norms}

Now let us use (5.8) to calculate norms $\left\{\lambda^{c}\right\}=\left\{\lambda^{B}\right\}=\{\lambda\}$,

$$
\left\langle 0\left|\prod_{j=1}^{N} C\left(\lambda_{j}\right) \prod_{j=1}^{N} B\left(\lambda_{j}\right)\right| 0\right\rangle=\prod_{j>k} g\left(\lambda_{j}, \lambda_{k}\right) g\left(\lambda_{k}, \lambda_{j}\right)\left\langle 0\left|\operatorname{det}_{N} S\right| 0\right\rangle .
$$


In this case matrix $S$ looks like:

$$
\begin{gathered}
S_{j k}=\frac{g_{j k}}{h_{j k}} e^{\Phi_{A}\left(\lambda_{j}\right)+\Phi_{D}\left(\lambda_{k}\right)}+\frac{g_{k j}}{h_{k j}} e^{\Phi_{A}\left(\lambda_{k}\right)+\Phi_{D}\left(\lambda_{J}\right)} ; \quad j \neq k, \\
g_{j k} \equiv g\left(\lambda_{j} \lambda_{k}\right), \quad h_{j k}=h\left(\lambda_{j} \lambda_{k}\right), \\
S_{j j}=e^{\Phi_{A}\left(\lambda_{j}\right)+\Phi_{D}\left(\lambda_{J}\right)}\left\{i c \frac{\partial}{\partial \lambda_{j}}\left(\Phi_{A}\left(\lambda_{j}\right)-\Phi_{D}\left(\lambda_{j}\right)\right)-2\right\} .
\end{gathered}
$$

Let us move all factors $\exp \left(\Phi_{A}\right)$ and $\exp \left(\Phi_{D}\right)$ to the left, so $\operatorname{det}_{N} S$ will be represented in the form

$$
\operatorname{det}_{N} S=\prod_{j=1}^{N} e^{\Phi_{A}\left(\lambda_{j}\right)+\Phi_{D}\left(\lambda_{j}\right)} \operatorname{det}_{N} S .
$$

Matrix $s$ can be written in terms of the field

$$
\begin{gathered}
\varphi(\lambda)=\Phi_{A}(\lambda)-\Phi_{D}(\lambda)=q(\lambda)+p(\lambda), \\
q(\lambda)=q_{A}(\lambda)-q_{D}(\lambda), \quad p(\lambda)=\pi_{D}(\lambda)-\pi_{A}(\lambda)
\end{gathered}
$$

only. It is equal to:

$$
\begin{gathered}
s_{j k}=\frac{g_{j k}}{h_{j k}}+\frac{g_{k j}}{h_{k j}} \exp \left\{\varphi\left(\lambda_{k}\right)-\varphi\left(\lambda_{j}\right)\right\} ; \quad k \neq j ; \\
s_{j j}=\left.i c \frac{\partial}{\partial \lambda} \varphi(\lambda)\right|_{\lambda=\lambda_{J}}-2 ; \quad g_{j k}=g\left(\lambda_{j} \lambda_{k}\right)=\frac{i c}{\lambda_{j}-\lambda_{k}} .
\end{gathered}
$$

The result of the action of the square brackets in (6.4) to dual vacuum is

$$
\langle 0| \prod_{j=1}^{N} e^{\Phi_{A}\left(\lambda_{j}\right)+\Phi_{D}\left(\lambda_{j}\right)}=\left[\prod_{j=1}^{N} a\left(\lambda_{j}\right) d\left(\lambda_{j}\right)\right] \prod_{j, k=1}^{N} h\left(\lambda_{j} \lambda_{k}\right) \cdot\langle\tilde{0}| .
$$

Here

$$
\langle\tilde{0}|=\langle 0| \prod_{j=1}^{N} \exp \left\{\pi_{D}\left(\lambda_{j}\right)+\pi_{A}\left(\lambda_{j}\right)\right\} .
$$

Now let us separate from $\varphi(\lambda)$ its vacuum mean value

$$
\varphi(\lambda) \equiv\langle\tilde{0}|\varphi(\lambda)| 0\rangle+\varphi^{0}(\lambda) .
$$

Elementary calculations show that

$$
\langle\widetilde{0}|\varphi(\lambda)| 0\rangle=\langle\widetilde{0}|q(\lambda)| 0\rangle=\ln \frac{a(\lambda)}{d(\lambda)}+\sum_{n=1}^{N} \ln \frac{h\left(\lambda, \lambda_{n}\right)}{h\left(\lambda_{n}, \lambda\right)} .
$$

So we have for $\varphi^{0}(\lambda)$ :

$$
\begin{gathered}
\varphi^{0}(\lambda)=q^{0}(\lambda)+p^{0}(\lambda) ; \quad p^{0}(\lambda)|0\rangle=0=\langle\tilde{0}| q^{0}(\lambda), \\
{\left[p^{0}(\lambda), q^{0}(\mu)\right]=-\ln \{h(\lambda, \mu) h(\mu, \lambda)\} \equiv H(\lambda, \mu)=\ln \left(\frac{c^{2}}{c^{2}+(\lambda-\mu)^{2}}\right),} \\
{\left[p^{0}(\lambda), p^{0}(\mu)\right]=0 ; \quad\left[q^{0}(\lambda), q^{0}(\mu)\right]=0=\left[\varphi^{0}(\lambda), \varphi^{0}(\mu)\right] .}
\end{gathered}
$$


Later it will be useful to consider the integral operator $\mathbf{H}$ with the kernel $H(\lambda, \mu)$. So we have for norms:

$$
\left\langle 0\left|\prod_{j=1}^{N} C\left(\lambda_{j}\right) \prod_{j=1}^{N} B\left(\lambda_{j}\right)\right| 0\right\rangle=\left\{\prod_{j=1}^{N} a\left(\lambda_{j}\right) d\left(\lambda_{j}\right)\right\} \prod_{j \neq k} f\left(\lambda_{j}, \lambda_{k}\right)\left\langle\tilde{0}\left|\operatorname{det}_{N} s\right| 0\right\rangle .
$$

Matrix elements of $s$ is equal to:

$$
s_{j j}=\left.i c \frac{\partial}{\partial \lambda}\left[\ln \frac{a(\lambda)}{d(\lambda)}+\sum_{k=1}^{N} \ln \frac{h\left(\lambda, \lambda_{k}\right)}{h\left(\lambda_{k}, \lambda\right)}\right]\right|_{\lambda=\lambda_{j}}-2+\left.i c \frac{\partial \varphi^{0}(\lambda)}{\partial \lambda}\right|_{\lambda=\lambda_{j}}
$$

in the case $j \neq k$ we have:

$$
S_{j k}=\frac{g_{j k}}{h_{j k}}+\frac{g_{k j}}{h_{k j}} e^{\varphi^{0}\left(\lambda_{k}\right)-\varphi^{0}\left(\lambda_{J}\right)} \times \frac{a\left(\lambda_{k}\right)}{d\left(\lambda_{k}\right)} \frac{d\left(\lambda_{j}\right)}{a\left(\lambda_{j}\right)} \prod_{n=1}^{N} \frac{h\left(\lambda_{k} \lambda_{n}\right)}{h\left(\lambda_{n} \lambda_{k}\right)} \frac{h\left(\lambda_{n} \lambda_{j}\right)}{h\left(\lambda_{j} \lambda_{n}\right)} .
$$

Remark. The problem of evaluation of $\left\langle\tilde{0}\left|\operatorname{det}_{N} s\right| 0\right\rangle$ is the problem of normal ordering. One can use standard methods of quantum field theory to evaluate this mean value. In the expression for $\operatorname{det}_{N} s$ let us replace quantum field $\varphi^{0}(\lambda)$ by complex function $\varphi^{0}(\lambda)$, then the mean value of $\operatorname{det}_{N} s$ in (6.14) can be represented as a functional integral:

$$
\int \prod d \varphi^{0}(\lambda) \operatorname{det}_{N} s\left[\varphi^{0}(\lambda)\right] \exp \left\{-\frac{1}{2} \int d \lambda d \mu \varphi^{0}(\lambda)\left(H^{-1}\right)(\lambda, \mu) \varphi^{0}(\mu)\right\}
$$

Here $H^{-1}(\lambda, \mu)$ is the kernel of operator inverse to $\mathbf{H}$.

Now let us study the norms of the eigenfunctions of transfer matrix $\tau(\mu)$. Equation (3.16) permits us to reduce expression (6.16). Finally we have:

$$
\left\langle 0\left|\prod_{j=1}^{N} C\left(\lambda_{j}\right) \prod_{j=1}^{N} B\left(\lambda_{j}\right)\right| 0\right\rangle=\left\{\prod_{j=1}^{N} a\left(\lambda_{j}\right) d\left(\lambda_{j}\right)\right\} \prod_{j \neq k} f\left(\lambda_{j} \lambda_{k}\right)\langle\tilde{0}|\operatorname{det} \mathbf{n}| 0\rangle .
$$

Matrix $\mathbf{n}$ is equal to:

$$
\begin{aligned}
\mathbf{n}_{j k}= & i c \delta_{k j} \frac{\partial}{\partial \lambda_{j}}\left[\ln \frac{a\left(\lambda_{j}\right)}{d\left(\lambda_{j}\right)}+\sum_{n=1}^{N} \ln \frac{h\left(\lambda_{j}, \lambda_{n}\right)}{h\left(\lambda_{n}, \lambda_{j}\right)}\right] \\
& +\frac{g_{j k}}{h_{j k}}+\frac{g_{k j}}{h_{k j}} \exp \left\{\varphi^{0}\left(\lambda_{k}\right)-\varphi^{0}\left(\lambda_{j}\right)\right\} .
\end{aligned}
$$

Detailed analysis [2] shows that $\varphi^{0}$ in (6.19) can be replaced by zero

$$
\operatorname{det}\left(i c \delta_{k j} \frac{\partial}{\partial \lambda_{j}}\left[\ln \frac{a\left(\lambda_{j}\right)}{d\left(\lambda_{j}\right)}+\sum_{n=1}^{N} \ln \frac{h\left(\lambda_{j}, \lambda_{n}\right)}{h\left(\lambda_{n}, \lambda_{j}\right)}\right]-\frac{2 c^{2}}{c^{2}+\lambda_{j k}^{2}}\right)=\langle\tilde{0}|\operatorname{det} \mathbf{n}| 0\rangle .
$$

\section{Thermodynamic Limit}

In the thermodynamic limit the number of particles $N$ and the length of the box $L$ go to infinity, but the density $D=N / L$ is fixed. The simplest way to describe this is to go back to the nonlinear Schrödinger equation from two-site generalised model (3.18)-(3.20). In the zero temperature case in the ground state parameters $\lambda_{j}$ are condensed $\left(\lambda_{j+1}-\lambda_{j}\right) \sim O(1 / L)$. It is convenient to introduce the distribution 
function $\varrho(\lambda)$ of the particles in the momentum space

$$
\varrho\left(\lambda_{j}\right)=1 / L\left(\lambda_{j+1}-\lambda_{j}\right) .
$$

The system of transcendental equations (3.16) turns into the Lieb integral equation,

$$
\varrho(\lambda)-\frac{1}{2 \pi} \int_{-q}^{q} K(\lambda, \mu) \varrho(\mu)=\frac{1}{2 \pi} ; \quad K(\lambda, \mu)=\frac{2 c}{c^{2}+(\hat{\lambda}-\mu)^{2}} .
$$

Here $q$ is Fermi momentum, and

$$
D=\int_{-q}^{q} \varrho(\lambda) d \lambda .
$$

In the case of finite temperature the model is described by two distribution functions $\varrho_{h}$ and $\varrho_{p}$ (the distribution of particles and holes). The most important is their ratio, which is denoted by

$$
\varrho_{h}(\lambda) / \varrho_{p}(\lambda)=\exp \{\varepsilon(\lambda) / T\} .
$$

It is defined by the Yang integral equation:

$$
\varepsilon(\lambda)=\lambda^{2}-h-\frac{T}{2 \pi} \int_{-\infty}^{\infty} K(\lambda, \mu) \ln [1+\exp \{-\varepsilon(\mu) / T\}] d \mu .
$$

In the papers $[6,8]$ it is shown that in the frame of the Bethe Ansatz there exists a very simple rule to obtain an expression of some value in the finite temperature case. It is enough to take the expression of this value for the zero temperature case and replace the measure of integration:

$$
\int_{-q}^{q} d \lambda \cdot \rightarrow \int_{-\infty}^{\infty} \frac{d \lambda}{(1+\exp \{\varepsilon(\lambda) / T\})}
$$

Now let us discuss the thermodynamic limit of norms of eigenfunctions (6.18) for zero temperature. Direct calculations show that

$$
\frac{\left\langle 0\left|\prod_{j=1}^{N} C\left(\lambda_{j}\right) \prod_{j=1}^{N} B\left(\lambda_{j}\right)\right| 0\right\rangle}{\left[\prod_{j \neq k} f\left(\lambda_{j} \lambda_{k}\right)\right]\left[\prod_{j=1}^{N} a\left(\lambda_{j}\right) d\left(\lambda_{j}\right) \cdot c \cdot 2 \pi L \varrho\left(\lambda_{j}\right)\right]}=\langle\tilde{0}|\operatorname{det}(1+\mathbf{N})| 0\rangle .
$$

Here $\mathbf{N}$ is an integral operator on the interval $[-q, q]$ with the kernel

$$
N(\lambda, \mu)=\left(\frac{c}{\lambda-\mu}\right) \frac{1}{2 \pi}\left(\frac{e^{\varphi^{0}(\mu)-\varphi^{0}(\lambda)}}{\mu-\lambda+i c}-\frac{1}{\lambda-\mu+i c}\right) .
$$

As the commutator $\left[p^{0}(\lambda), q^{0}(\mu)\right](6.12)$ vanishes at $\lambda \rightarrow \mu$, the kernel (7.7) is nonsingular and there are no divergences. Using (6.20) it is clear that

$$
\begin{gathered}
\langle\tilde{0}|\operatorname{det}(1+\mathbf{N})| 0\rangle=\operatorname{det}\left(1-\frac{1}{2 \pi} \mathbf{K}\right), \\
K(\lambda, \mu)=2 c /\left[c^{2}+(\lambda-\mu)^{2}\right] .
\end{gathered}
$$


In the finite temperature case the operator $\mathbf{N}$ in (7.6) is replaced by the integral operator on the real axis with the kernel

$$
N_{T}(\lambda, \mu)=\frac{N(\lambda, \mu)}{1+\exp \{\varepsilon(\mu) / T\}} .
$$

Exploitation of (6.20) shows that

$$
\begin{gathered}
\left\langle\tilde{0}\left|\operatorname{det}\left(1+\mathbf{N}_{T}\right)\right| 0\right\rangle=\operatorname{det}\left(1-\frac{1}{2 \pi} \mathbf{K}_{T}\right), \\
K_{T}(\lambda, \mu)=\frac{K(\lambda, \mu)}{1+\exp \{\varepsilon(\mu) / T\}} .
\end{gathered}
$$

\section{Operators $\Omega$}

The main aim of this paper is to calculate the generating functional of correlators. It is equal to the mean value of $A_{1}(\mu)$ with respect to the eigenfunction of the transfer matrix [see (2.10)]

$$
\begin{aligned}
& \left\langle 0\left|\prod_{j=1}^{N} C\left(\lambda_{j}\right) A_{1}(\mu) \prod_{j=1}^{N} B\left(\lambda_{j}\right)\right| 0\right\rangle^{I}, \\
& \frac{a^{I}\left(\lambda_{j}\right)}{d^{I}\left(\lambda_{j}\right)} \prod_{j=1}^{N} \frac{h\left(\lambda_{j} \lambda_{n}\right)}{h\left(\lambda_{n} \lambda_{j}\right)}=(-1)^{N-1} .
\end{aligned}
$$

Compare with (4.3). We shall introduce a more general object $M_{N}$ which has the recursion formula similar to (4.6). The mean value (8.1) will be a special case of $M_{N}$. Define two operators $\Omega_{1}^{B}$ and $\Omega_{2}^{B}$ acting to the right. Their action on the basis (3.2), (3.3) is by definition:

$$
\begin{aligned}
& \Omega_{1}^{B} \prod_{j=1}^{N} B_{1}\left(\lambda_{j}\right)|0\rangle_{1}=\prod_{j=1}^{N} \omega_{1}^{B}\left(\lambda_{j}\right) \prod_{j=1}^{N} B_{1}\left(\lambda_{j}\right)|0\rangle_{1}, \\
& \Omega_{2}^{B} \prod_{j=1}^{N} B_{2}\left(\lambda_{j}\right)|0\rangle_{2}=\prod_{j=1}^{N} \omega_{2}^{B}\left(\lambda_{j}\right) \prod_{j=1}^{N} B_{2}\left(\lambda_{j}\right)|0\rangle_{2} .
\end{aligned}
$$

Here $\omega_{1,2}^{B}$ are two complex valued functions. Define now two other operators $\Omega_{1}^{c}$ and $\Omega_{2}^{c}$ acting to the left. Their action on the dual basis is by definition:

$$
\begin{aligned}
& { }_{1}\langle 0| \prod_{j=1}^{N} C_{1}\left(\lambda_{j}\right) \Omega_{1}^{c}=\prod_{j=1}^{N} \omega_{1}^{c}\left(\lambda_{j}\right)_{1}\langle 0| \prod_{j=1}^{N} C_{1}\left(\lambda_{j}\right), \\
& { }_{2}\langle 0| \prod_{j=1}^{N} C_{2}\left(\lambda_{j}\right) \Omega_{2}^{c}=\prod_{j=1}^{N} \omega_{2}^{c}\left(\lambda_{j}\right)_{2}\langle 0| \prod_{j=1}^{N} C_{2}\left(\lambda_{j}\right) .
\end{aligned}
$$

Here $\omega_{1}^{c}(\lambda)$ and $\omega_{2}^{c}(\lambda)$ are two other complex functions. By means of $(3.13),(3.14)$ the matrix element

$$
M_{N}=\left\langle 0\left|\prod_{j=1}^{N} C\left(\lambda_{j}^{c}\right) \Omega_{1}^{c} \Omega_{2}^{c} \Omega_{2}^{B} \Omega_{1}^{B} \prod_{j=1}^{N} B\left(\lambda_{j}^{B}\right)\right| 0\right\rangle
$$


can be written in the form:

$$
\begin{aligned}
M_{N}= & \sum_{1}\left\langle 0\left|\prod_{\mathrm{I}} C_{1}\left(\lambda_{\mathrm{I}}^{c}\right) \prod_{\mathrm{I}} B_{1}\left(\lambda_{\mathrm{I}}^{B}\right)\right| 0\right\rangle_{1}\left\langle 0\left|\prod_{\mathrm{II}} C_{2}\left(\lambda_{\mathrm{II}}^{c}\right) \prod_{\mathrm{II}} B_{2}\left(\lambda_{\mathrm{II}}^{B}\right)\right| 0\right\rangle_{2} \\
& \times \prod_{\mathrm{I}} \omega_{1}^{c}\left(\lambda_{\mathrm{I}}^{c}\right) \omega_{1}^{B}\left(\lambda_{\mathrm{I}}^{B}\right) a_{2}\left(\lambda_{\mathrm{I}}^{B}\right) d_{2}\left(\lambda_{\mathrm{I}}^{c}\right) \\
& \prod_{\mathrm{II}} \omega_{2}^{c}\left(\lambda_{\mathrm{II}}^{c}\right) \omega_{2}^{B}\left(\lambda_{\mathrm{II}}^{B}\right) a_{1}\left(\lambda_{\mathrm{II}}^{c}\right) d_{1}\left(\lambda_{\mathrm{II}}^{B}\right) \\
& \times \prod_{\mathrm{I}} \prod_{\mathrm{II}} f\left(\lambda_{\mathrm{I}}^{B}, \lambda_{\mathrm{II}}^{B}\right) f\left(\lambda_{\mathrm{II}}^{c}, \lambda_{\mathrm{I}}^{c}\right) .
\end{aligned}
$$

Here summation is over partition of the set $\left\{\lambda^{B}\right\}=\left\{\lambda_{\mathrm{I}}^{B}\right\} \cup\left\{\lambda_{\mathrm{II}}^{B}\right\}$ and independent partition of the set $\left\{\lambda^{c}\right\}=\left\{\lambda_{\mathrm{I}}^{c}\right\} \cup\left\{\lambda_{\text {II }}^{c}\right\}$ with one condition $\#\left\{\lambda_{I}^{c}\right\}=\#\left\{\lambda_{\mathrm{I}}^{B}\right\}$. Formula (8.6) represents $M_{N}$ in terms of scalar products. It can be used as a definition of $M_{N}$.

It is useful to recall that $M_{N}$ depends on eight arbitrary functions,

$$
M_{N}=M_{N}\left(\left[\omega_{1,2}^{B, c}(\lambda), a_{1,2}(\lambda), d_{1,2}(\lambda)\right]\right) .
$$

By means of (4.5) one can see that the matrix element of $A_{1}(\mu)$ is the special case of $M_{N}[\operatorname{see}(3.15)]$,

$$
\left\langle 0\left|\prod_{j=1}^{N} C\left(\lambda_{j}^{c}\right) A_{1}(\mu) \prod_{j=1}^{N} B\left(\lambda_{j}^{B}\right)\right| 0\right\rangle^{I}=a_{1}(\mu)\left\langle 0\left|\prod_{j=1}^{N} C\left(\lambda_{j}^{c}\right) \Omega_{1}^{c} \Omega_{2}^{c} \Omega_{2}^{B} \Omega_{1}^{B} \prod_{j=1}^{N} B\left(\lambda_{j}^{B}\right)\right| 0\right\rangle .
$$

The left-hand side depends on arbitrary functions with the index $I\left(d_{1}^{I}(\mu)=0\right)$. The arbitrary functions of the right-hand side are equal to

$$
\begin{gathered}
\omega_{1}^{B}=\omega_{1}^{c}=\omega_{2}^{c}=1 ; \quad \omega_{2}^{B}(\lambda)=f^{-1}(\mu, \lambda) ; \\
a_{1}(\lambda)=a_{1}^{I}(\lambda) ; \quad a_{2}(\lambda)=a_{2}^{I}(\lambda) ; \quad d_{2}(\lambda)=d_{2}^{I}(\lambda), \\
d_{1}(\lambda)=d_{1}^{I}(\lambda) f(\mu, \lambda) .
\end{gathered}
$$

\section{Generating Functional of Correlators}

The program of Sect. 4-7 can be done for $M_{N}(8.6)$. In the thermodynamic limit it is equal to the determinant similar to (7.6). Formula (8.8) permits us to calculate the mean value of $A_{1}(\mu)$, which is the generating functional of correlators for the nonlinear Schrödinger equation [at $\mu \rightarrow i \infty$, see (2.9) and (2.10)]. [We need asymptotic decomposition of $\left\langle A_{1}(\mu)\right\rangle / a_{1}(\mu)$ in $1 / \mu$.] The answer for the thermodynamic limit of $\left\langle A_{1}(\mu)\right\rangle$ [see (8.1), (8.2)] is:

$$
\frac{\left\langle 0\left|\prod_{j=1}^{N} C\left(\lambda_{j}\right) A_{1}(\mu) \prod_{j=1}^{N} B\left(\lambda_{j}\right)\right| 0\right\rangle}{a_{1}(\mu)\left\langle 0\left|\prod_{j=1}^{N} C\left(\lambda_{j}\right) \prod_{j=1}^{N} B\left(\lambda_{j}\right)\right| 0\right\rangle}=\frac{\langle 0|\operatorname{det}(1+\mathbf{V})| 0\rangle}{\operatorname{det}\left(1-\frac{1}{2 \pi} \mathbf{K}\right)^{-}} .
$$

The denominator in the right-hand side is defined by formula (7.8). In the numerator $\mathbf{V}$ is the integral operator on the segment $[-q, q]$ with the kernel 
$V\left(\lambda_{1}, \lambda_{2}\right):$

$$
\begin{aligned}
2 \pi c V\left(\lambda_{1}, \lambda_{2}\right)= & \frac{g\left(\lambda_{1} \lambda_{2}\right)}{h\left(\lambda_{1} \lambda_{2}\right)}+\frac{g\left(\lambda_{2} \lambda_{1}\right)}{h\left(\lambda_{2} \lambda_{1}\right)} e^{i x\left(\lambda_{2}-\lambda_{1}\right)} \times e^{\varphi_{62}\left(\lambda_{2}\right)-\varphi_{62}\left(\lambda_{1}\right)} \\
& +f\left(\mu, \lambda_{1}\right) e^{\varphi_{31}\left(\lambda_{1}\right)} e^{\varphi_{42}\left(\lambda_{2}\right)} \times\left[\frac{g\left(\lambda_{2}, \lambda_{1}\right)}{h\left(\lambda_{2}, \lambda_{1}\right)}+\frac{g\left(\lambda_{1} \lambda_{2}\right)}{h\left(\lambda_{1} \lambda_{2}\right)} e^{i x\left(\lambda_{2}-\lambda_{1}\right)}\right. \\
& \left.\times \frac{f\left(\mu, \lambda_{2}\right)}{f\left(\mu, \lambda_{1}\right)} \times \exp \left\{\varphi_{73}\left(\lambda_{1}\right)-\varphi_{73}\left(\lambda_{2}\right)\right\}\right]
\end{aligned}
$$

It depends on four quantum fields $\varphi_{a}$ where the index $a$ runs through four values $a=62 ; 73 ; 31 ; 42$. Each field can be represented in the form

$$
\varphi_{a}(\lambda)=q_{a}(\lambda)+p_{a}(\lambda) .
$$

They act on the vacuum as follows:

$$
p_{a}(\lambda)|0\rangle=0 ; \quad\langle 0| q_{a}(\lambda)=0 .
$$

Commutation relations are:

$$
\begin{aligned}
& {\left[p_{62}(\lambda), q_{62}(\mu)\right]=H(\lambda, \mu)=\ln \left(\frac{c^{2}}{c^{2}+(\lambda-\mu)^{2}}\right)=-\ln \{h(\lambda, \mu) h(\mu, \lambda)\},} \\
& {\left[p_{73}(\lambda), q_{73}(\mu)\right]=H(\lambda, \mu),} \\
& {\left[p_{31}(\lambda), q_{31}(\mu)\right]=H(\lambda, \mu),} \\
& {\left[p_{42}(\lambda), q_{42}(\mu)\right]=H(\lambda, \mu),} \\
& {\left[p_{62}(\lambda), q_{73}(\mu)\right]=0=\left[p_{73}(\mu), q_{62}(\lambda)\right],} \\
& {\left[p_{62}(\lambda), q_{31}(\mu)\right]=\ln h(\mu, \lambda)=\left[p_{31}(\mu), q_{62}(\lambda)\right],} \\
& {\left[p_{62}(\lambda), q_{42}(\mu)\right]=-\ln h(\lambda, \mu)=\left[p_{42}(\mu), q_{62}(\lambda)\right],} \\
& {\left[p_{73}(\lambda), q_{31}(\mu)\right]=\ln h(\lambda, \mu)=\left[p_{31}(\mu), q_{73}(\lambda)\right],} \\
& {\left[p_{73}(\lambda), q_{42}(\mu)\right]=-\ln h(\mu, \lambda)=\left[p_{42}(\mu), q_{73}(\lambda)\right],} \\
& {\left[p_{31}(\lambda), q_{42}(\mu)\right]=-H(\lambda, \mu)=\left[p_{42}(\mu), q_{31}(\lambda)\right] .}
\end{aligned}
$$

One should emphasize that all four fields $\varphi_{a}$ commute,

$$
\left[\varphi_{a}(\lambda), \varphi_{b}(\mu)\right]=0, \quad a, b=62 ; 73 ; 31 ; 42 .
$$

Formula (9.1) is the main result of the paper. It permits us to calculate correlators of the model in terms of the auxiliary quantum field theory. The fact that the quantum fields $\varphi_{a}$ commute permits us to transform the determinant as a classical one. It is convenient to analyze asymptotics of correlators at large distances.

Finally I would like to mention that everything simplifies at $c \rightarrow \infty$. In this case all commutators are equal to zero and

$$
\begin{gathered}
\langle\tilde{0}|\operatorname{det}(1+\mathbf{V})| 0\rangle=\operatorname{det}\left(1+\mathbf{V}^{0}\right), \\
V^{0}\left(\lambda_{1}, \lambda_{2}\right)=\frac{c}{2 \pi\left(\lambda_{1}-\lambda_{2}\right)}\left(\frac{1}{\mu-\lambda_{1}}+\frac{\exp \left\{i x\left(\lambda_{2}-\lambda_{1}\right)\right\}}{\lambda_{2}-\mu}\right) .
\end{gathered}
$$


The asymptotic expansion of $\operatorname{det}\left(1+\mathbf{V}^{0}\right)$ at $\mu \rightarrow i \infty$ is direct. Using trace identities one obtains the correct result (2.11):

$$
\left\langle\psi^{+}(x) \psi(x) \psi^{+}(0) \psi(0)\right\rangle=\left(\frac{q}{\pi}\right)^{2}-\left(\frac{\sin q x}{\pi x}\right)^{2} ; \quad D=\frac{q}{\pi} .
$$

At finite temperature, $\mathbf{V}$ in (9.1) is replaced by $\mathbf{V}_{T}$ (this is the integral operator on the real axis). Its kernel is equal to

$$
V_{T}\left(\lambda_{1}, \lambda_{2}\right)=V\left(\lambda_{1}, \lambda_{2}\right) /\left(1+\exp \left\{\varepsilon\left(\lambda_{2}\right) / T\right\}\right) .
$$

At $c=\infty$ we obtain the correct result:

$$
\left\langle\psi^{+}(x) \psi(x) \psi^{+}(0) \psi(0)\right\rangle=D^{2}-\frac{1}{4 \pi^{2}}\left[\int_{-\infty}^{\infty} \frac{e^{i x \lambda} d x}{1+\exp \left\{\left(\lambda^{2}-h\right) / T\right\}}\right]^{2} .
$$

I would like to mention that ideas of papers [2-6] were developed also in a different direction. F. A. Smirnov and A. N. Kirilov have calculated the senior Fourier coefficient of the form factor.

Acknowledgements. I would like to thank A. G. Izergin for discussions and the International Centre for Theoretical Physics for hospitality.

\section{References}

1. Faddeev, L.D.: Quantum inverse scattering method. Sov. Sci. Rev. Math. Phys. C1, 107-166 (1981)

2. Korepin, V.E.: Calculation of norms of Bethe wave functions. Commun. Math. Phys. 86, 319-418 (1982)

3. Korepin, V.E.: Analysis of bilinear relation for 6-vertex model. Doklady AN SSSR 265, 1361-1364 (1982)

4. Izergin, A.G., Korepin, V.E.: The quantum inverse scattering method approach to correlation functions. Commun. Math. Phys. 94, 67-92 (1984)

5. Korepin, V.E.: Correlation functions of the one-dimensional Bose gas. Commun. Math. Phys. 94, 93-113 (1984)

6. Bogoliubov, N.M., Izergin, A.G., Korepin, V.E.: Exactly solvable problems in condensed matter and relativistic field theory. Lecture Notes in Physics, Vol. 242, pp. 220-316. Berlin, Heidelberg, New York: Springer 1985

7. Izergin, A.G.: Partition function of 6 vertex model. Doklady AN SSSR (submitted)

8. Bogoliubov, N.M., Korepin, V.E.: Correlation length of the one-dimensional Bose gas. Nucl. Phys. B 257 [FS 114], 760-778 (1985)

Communicated by A. Jaffe

Received July 27, 1987 